\title{
ENZYME-HISTOCHEMICAL INVESTIGATIONS ON THE LYMPHATICS OF THE INTRINSIC UTERINE WALL IN THE MONKEY
}

\author{
RUi-CHENG Ji \\ Department of Anatomy, Oita Medical University, Oita 879-5593, Japan
}

\begin{abstract}
Topographic and ultrastructural characteristics in the monkey uterine lymphatics were demonstrated in the separate phases (proliferative, secretory and menstrual) of the sexual cycle and during pregnancy with 5'-nucleotidase (5'-Nase) and alkaline phosphatase (ALPase) staining methods. In the early proliferative phase, the newly forming and growing endometrial lymphatics with very irregular endothelial outlines were quite sparse and small in diameter $(18-23 \mu \mathrm{m})$, showing extremely weak 5'Nase activity. The lymphatics in the secretory phase extended as far as the middle endometrium, and their endothelium obviously protruded into both the luminal and basal aspects. Abundant mitochondria and pinocytotic vesicles appeared in the cytoplasm. The most striking finding was that initial lymphatics in the middle endometrium are affected by sporadic episodes of cessation of blood supply, exhibiting a partial destruction of the endothelial wall during menstruation through which lymph fluid and numerous lymphocytes escape from the devitalized lymphatics into surrounding connective tissue. During pregnancy, lymphatic networks with high 5'-Nase activity in the endo-myometrial interface and myometrium were well developed and greatly enlarged, in contrast to those of the nulliparous monkey. Lymphatic endothelium with notable protrusions showed typical overlapping, interdigitating intercellular junctions and intraendothelial channels. The results indicated that uterine lymphatics are involved in the total processes of the sexual cycle, especially in the endometrium. Their continuous increment of the volumetric density and morphological changes during pregnancy may correspond to intensive growth of the fetus and prompt modification of the tissue metabolism, in cooperation with hemochorial relation between the mother and fetus.
\end{abstract}

Morphological studies of the uterine lymph drainage have predominantly been carried out by traditional injection techniques to demonstrate lymphatic gross contour and connexion between the ipsilateral ovary and uterus in the sheep $(1,14)$, rabbit (17) and flying foxes (19). These studies had shown that the lymphatic plexus occurs limitedly at the endo-myometrial interface, but never extends into the endometrium. It has been

Correspondence to: Dr R.C. Ji at the above address. Tel \& Fax: ${ }^{+81-97-586-5623 ; ~ E-m a i l: ~ J I @ o i t a-m e d . a c . ~}$ jp believed that the efferent lymph drainage may be lacking in the endometrial layer in these mammalia $(17,22)$. In humans, however, a network of lymphatic capillaries was confirmed throughout the whole thickness of the endometrium by intratissue injection method (21). In rats, a few lymphatics were identified in the basal endometrium by using semithin sections stained with toluidine blue (5). These lymphatic capillaries were similar to those of the ovary, varying in size and shape cyclically in the ewe (3) and rabbit uterus after hCG injection (18).

Thus, the distribution of initial lymphatics in 
the uterine endometrium in mammalia and humans remains a matter of considerable debate. In addition, a rich lymphatic plexus in two rhesus monkeys was demonstrated by India ink injection at the junction of endometrium and myometrium, from which lymphatics ramified into the myometrium (27). A few other studies on the myometrial and perimetrial lymphatics $(7,17,23)$ revealed that prominent collecting vessels provided with valves occur in the loose connective tissue septum between the circular and longitudinal muscle layers or in the submesothelial layer. These valves mainly function as outlet-lamellae during lymphatic cycle. The functional morphology of the lymphatic circulation in the uterine wall remains to be clarified with particular reference to the sexual cycle and pregnancy (4). Detailed information about intrinsic uterine lymphatics is very important for understanding physiological feature and pathological occurrence of the female reproductive tract.

Investigations by using 5'-nucleotidase (5'Nase) and alkaline phosphatase (ALPase) enzymes have been proved as a reliable clue for studying intraorganic lymphatics and blood capillaries $(8,9,10,11,12,13)$. With regard to 5 '-Nase, a specific enzyme in dephosphorylating 5'-mononucleotides, it has widely been used as a marker for plasma membranes of numerous cell types. In the early experiment, 5'-Nase activity was also found strong in the lymphatic endothelium but obviously lower or lacking in the endothelium of blood capillaries, in contrast to ALPase whose activity is strong in the latter but either absent or extremely faint in the former (24). On the basis of the enzyme properties, 5'-Nase and ALPase were employed to differentiate between lymphatics and blood capillaries in the rat pharynx at the light microscopic level (26). More recently, 5'-Nase and ALPase staining techniques were successfully modified and improved to investigate the architecture of lymphatics and blood capillaries in combined light and electron microscopies $(12,13)$. Our recent reports, furthermore, showed that lymphatics in the developing phase or in the organs undergoing formidable volumetric variation, such as the urinary bladder, represent certain characteristics not only in the distribution and ultrastructure but also in the 5 -Nase activity $(9,11)$. In the present study, the 5'-Nase and ALPase staining techniques have been adopted to elucidate topography and fine structure of the uterine lymphatics. Results obtained gave us substantial informations as to cyclical dynamics of the lymphatics in the nongestational intrinsic uterine wall and to arrangement and characteristics of the lymphatics in the gestational uterine wall.

\section{MATERIALS AND METHODS}

Twelve Japanese monkeys (Macata fuscata) in various phases of the sexual cycle and during pregnancy were used. They were deeply anesthetized with ketamine $(10 \mathrm{mg} / \mathrm{kg}, i . m$.) and perfused via the thoracic aorta with cold formaldehyde $\mathrm{CaCl}_{2}$ fixative (4\% paraformaldehyde, $1 \% \mathrm{CaCl}_{2}$ ) in $0.1 \mathrm{M}$ cacodylate buffer containing $7 \%$ sucrose. Following laparotomy, the uterus was moved out from the abdomen. The tissue samples from the uterine corpus were frozen in OCT compound (Miles, USA) at $-80^{\circ} \mathrm{C}$. Frozen sections, $5-7 \mu \mathrm{m}$ thick, were cut with a cryostat at a cabinet temperature of $-18^{\circ} \mathrm{C}$. Changes of the sexual cycle in the non-gestational uterus mainly involve the lining endometrium of the corpus and may, for convenience, be divided into three phases: 1) menstrual, 2) proliferative, and 3) secretory. Therefore, the data on lymphatics was fundamentally assessed according to the different phases in the nulliparous monkey. 5'-Nase and ALPase staining methods were processed for identification of the lymphatics and blood vessels in all the specimens from the gestational and non-gestational uteri.

\section{5'-nucleotidase (5'-Nase) Activity for Lymphatics}

For light microscopy, the tissue sections were incubated in the 5 -Nase reactive medium $(8,12$, 25) with slight modification for $20 \mathrm{~min}$ at $37^{\circ} \mathrm{C}$, which mainly contains $2.9 \mathrm{mM}$ adenosine 5'monophosphate (AMP, Sigma, USA); 1.8 $\mathrm{mM} \mathrm{Pb}\left(\mathrm{NO}_{3}\right)_{2}$ and $2 \mathrm{mM}$ L-tetramisole. The samples were rinsed with $0.1 \mathrm{M}$ cacodylate buffer and immersed in $1 \%$ ammonium sulfide solution for 1-2 min at room temperature.

For scanning electron microscopy (backscattered electron imaging, BEI-SEM), the sections, incubated in the above-mentioned medium after light microscopic observation, were dehydrated in ascending concentrations of ethanol and treated with t-butyl alcohol freeze-drying method. Dried specimens were mounted on an aluminum stub and coated with carbon to prevent electron charging artifacts, then examined in a Hitachi S-800 
$\operatorname{SEM}(9,13)$.

For transmission electron microscopy (TEM), 5'-Nase cerium-based method of Robinson and Karnovsky (20) was used in these analyses (10). The medium chiefly consists of $1.0 \mathrm{mM}$ adenosine 5 '-monophosphate and $2.0 \mathrm{mM} \mathrm{CeCl} 3$. Tissue samples were incubated for $25-35 \mathrm{~min}$ at $37^{\circ} \mathrm{C}$. They were postfixed with $2 \%$ osmium tetroxide solution and uniformly dehydrated through graded concentrations of ethanol, and then embedded in Epok 812 (Oken, Tokyo, Japan). Ultrathin sections were double-contrasted with uranyl acetate and lead citrate, and examined under a 1,200 EX electron microscope.

\section{Alkaline Phosphatase (ALPase) Activity for Blood Vessels}

ALPase staining for light microscopic study of blood vessels was applied in some sections and whole mount preparations by using Azo-dye reactive medium, containing $40 \mathrm{mg}$ of naphthol AS-MX phosphate (Sigma, USA) dissolved in 2 $\mathrm{mL}$ of $\mathrm{N}, \mathrm{N}^{\prime}$-dimethylformamide and $40 \mathrm{mg}$ of fast blue $\mathrm{BB}$ salt dissolved in $40 \mathrm{~mL}$ of $0.1 \mathrm{M}$ Tris- $\mathrm{HCl}$ buffer $(\mathrm{pH} 8.5)$ for $40-50 \mathrm{~min}$ at $4^{\circ} \mathrm{C}(8$,
16). For 5'-Nase-ALPase double staining, ALPase reaction was usually followed by 5'-Nase procedure with a slightly extending time.

The following 5'-Nase and ALPase staining procedures were done for control groups, a) AMP or naphthol AS-MX phosphate was omitted; b) $50 \mathrm{mM} \mathrm{NiCl}_{2}$ or $5 \mathrm{mM}$ L-tetramisole was added to the reactive medium; c) the enzymes were inactivated at $60^{\circ} \mathrm{C}$ for $60 \mathrm{~min}$ before incubation.

\section{RESULTS \\ Lymphatics in the Non-gestational Uterus}

5'-Nase-positive lymphatics in the endometrium generally varied in number, arrangement and structure depending on the sexual cycle. In the early proliferative phase, initial lymphatics with irregular endothelial outlines were quite sparse and small in diameter $(18-23 \mu \mathrm{m})$, showing much lower 5'-Nase activity than those in the secretory phase (Fig. 1a). A few reactive products were scattered in the lymphatic endothelium. No 5'Nase reactive product was seen in the endothelium of blood capillaries. Slender endothelial wall possessed simple immature intercellular junctions. Cytoplasm was rich in free ribosomes

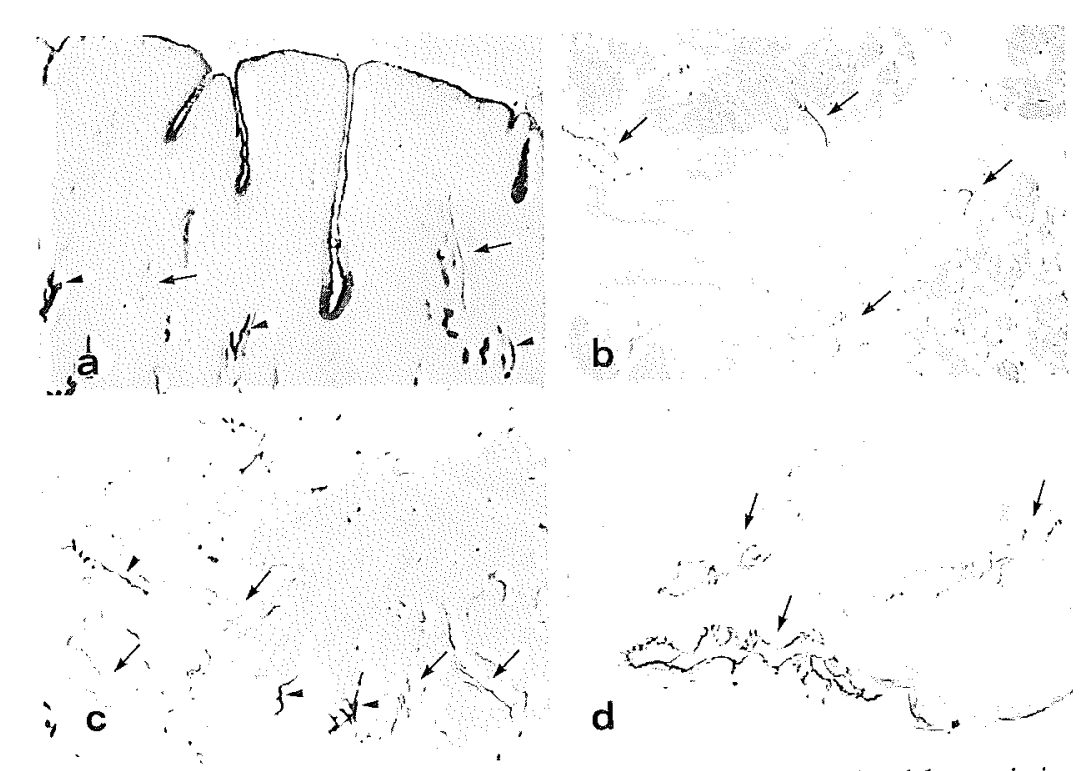

Fig. 1 Photomicrographs of the frozen sections 'with 5'-Nase-ALPase double staining (a, c) and 5'-Nase staining (b,d) showing 5'-Nase-positive lymphatics (arrows) and ALPase-positive blood vessels (arrowheads) in the different layers of the non-gestational uterine wall.

a. 5'-Nase-positive lymphatics are dispersively visualized in the endometrium of the early proliferative phase. $(\times 85)$

b, c. Numerous small lymphatics are distributed between the muscle bundles in the mid-late (b), and late (c)

proliferative phases. $(\times 35)$
d. Irregular lymphatics appear in the perimetrial connective tissue in the mid-late proliferative phase. $(\times 110)$ 
and rough endoplasmic reticula, but pinocytotic vesicles were rare, indicating the newly forming or growing initial lymphatics. Endothelial organelles usually resided in the perinuclear region. No typical anchoring filament and basal lamina could be found (Figs. $2 a$ and $3 a$ ). In the midproliferative phase, the lymphatics with irregular outline were more readily found at the endomyometrial interface. Dilatation of lymphatics (an average calibre of $37 \mu \mathrm{m}$ ) was more prominent in the mid-proliferative phase than those in the early phase (Fig. 2b), and reactive precipitates increased in number in the slender endothelial wall of lymphatics in the mid-proliferative phase (Fig. 3b). Lymphatics in the secretory phase extended as far as the middle endometrium and surrounded the glands and blood vessels. Only the most superficial layer was devoid of lymphatics. These lymphatics showed higher 5'-Nase activity in the secretory phase than in the proliferative and menstrual phases. Lymphatic endoth- elium was relatively thick and obviously protruded into both the luminal and basal aspects (Fig. 2c). Numerous mitochondria and pinocytotic vesicles $(60-80 \mathrm{~nm})$ appeared in the cytoplasm (Fig. 3c), exibiting a structural feature for edematous fluid absorption in the secretory phase. In the menstrual phase, initial lymphatics were slightly enlarged with an average calibre of $56 \mu \mathrm{m}$ and more irregular. The endothelium was attached with sparse 5'-Nase reactive granules. Occasionally, a partial destruction of initial lymphatics surrounding the uterine glands of the middle endometrium could be encountered (Figs. $2 \mathrm{~d}$ and $3 \mathrm{~d}$ ), through which lymph fluid and numerous lymphocytes escaped from the devitalized lymphatics into adjacent connective tissue.

Abundant 5'-Nase-positive initial lymphatics were seen between the muscle coats (Fig. 1, b and c), their endothelium was supported by many collagen fibers. In the early proliferative phase, initial lymphatics with flat profile were obviously

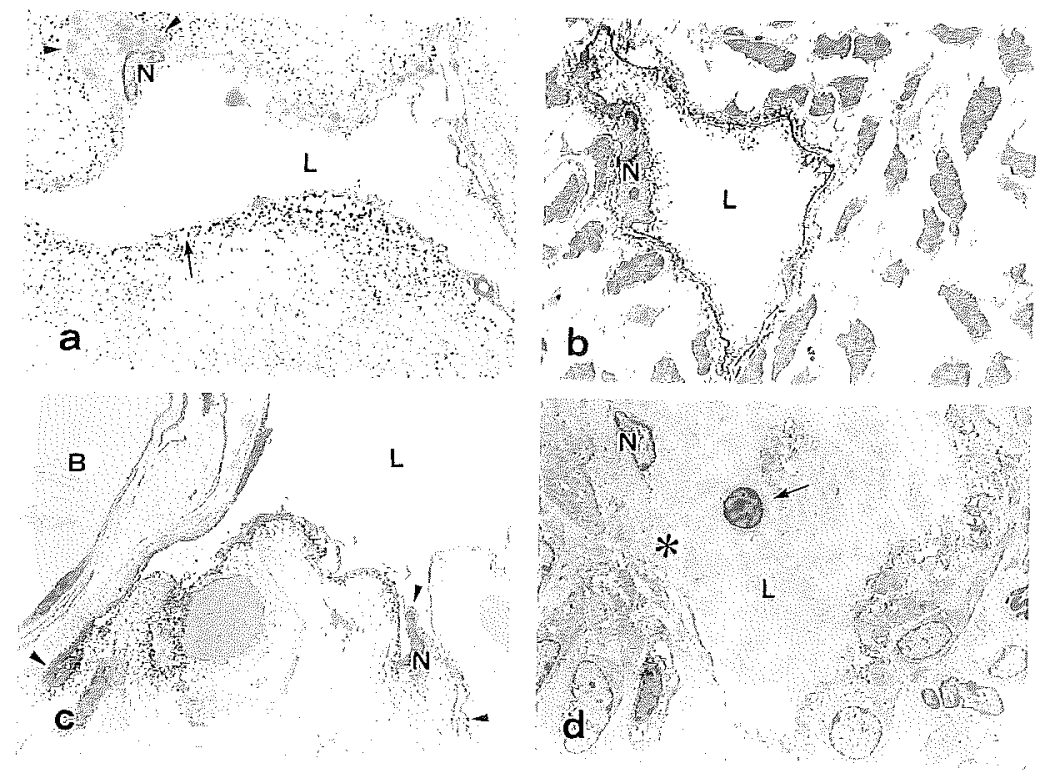

Fig. 2 Electron micrographs of 5'-Nase-positive lymphatics in the endometrium of the non-gestational uterine wall. L: lymphatics; B: blood vessel; N: nuclei of lymphatic endothelia a. In the early proliferative phase, an initial lymphatic has a weak 5'-Nase activity, exhibiting irregular attachment of reactive product. The endothelial wall represents a simple overlapping intercellular junction (arrow). Some endothelial organells reside in the perinuclear region (arrowheads). $(\times 5,000)$

b. A slightly dilated initial lymphatic with slender endothelial wall is shown in the mid-proliferative phase. $(\times 2,000)$

c. In the secretory phase, lymphatic endothelium with high 5'-Nase activity shows obvious protrusions into the luminal and basal surfaces (arrowheads). No reactive product is seen in the endothelium of the blood vessel. $(\times 2,000)$

d. An initial lymphatic endothelium shows a partial destruction (asterisk) in the middle endometrium of the early menstrual phase. A lymphocyte (arrow) and lymph are escaping from the devitalized endothelial wall into surrounding connective tissue. $(\times 1,500)$ 


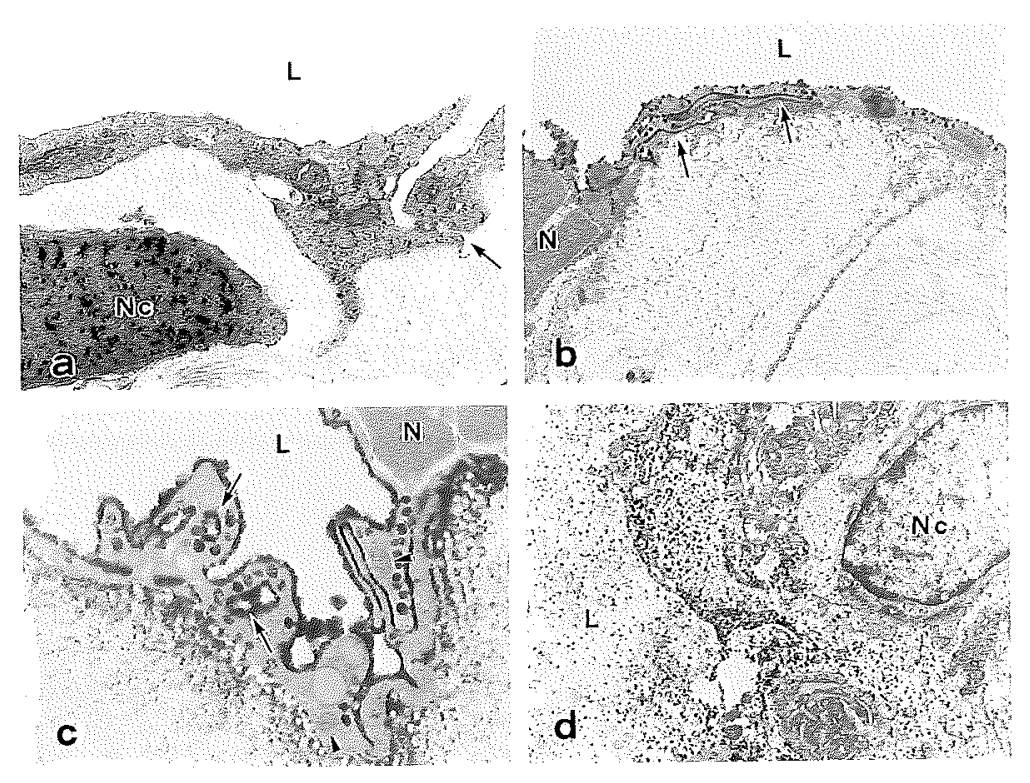

Fig. 3 Electron micrographs in higher magnification showing 5'-Nase-positive lymphatics in the endometrium of the non-gestational uterine wall. L: lymphatics; N: nuclei of lymphatic endothelia; Nc: nuclei of connective tissue cells

a. In the early proliferative phase, the endothelia of an initial lymphatic indicate extremely faint 5'-Nase activity and an end-to-end intercellular junction (arrow). Free ribosomes and rough endoplasmic reticula are seen in the cytoplasm. $(\times 24,000)$

b. More reactive granules are distributed in the slender endothelial wall in the mid-proliferative phase than in the early phase (3a). Arrows indicate a simple interdigitating junction. $(\times 13,000)$

c. In the secretory phase, the pinocytotic vesicles (arrows) and mitochondria (arrowheads) are shown in the high 5'-Nase-positive lymphatic endothelium. The vesicles are also lined by 5'-Nase reactive product. ( $X$ $25,000)$

d. Further magnification of Fig. $2 \mathrm{~d}$ showing the devitalized lymphatic endothelium which is attached with sparse 5'-Nase reactive precipitates. No basal lamina is seen around the lymphatic endothelium. $(\times 7,000)$

distinguished from 5'-Nase-negative arteriole and venule (Fig. 4a). In the mid-late proliferative phase, 5'-Nase reactive precipitates were evenly distributed in the luminal and basal surfaces of lymphatic endothelium (Fig. 4b). These lymphatics were larger than those of the early proliferative phase. Initial lymphatics with high 5'-Nase activity in the secretory phase were filled with rich lymph (Fig. 4c). Collecting lymphatics provided with many valves, occurred in the intramuscular connective tissue and possessed thicker endothelial walls. An abundance of collecting lymphatics usually further enhanced in the external longitudinal muscle layer, where they were sometimes surrounded by a fine network of blood capillaries. Three-dimensional two-flap valves were readily visualized as a strong highlight in the BEI-SEM observation (Fig. 4d).

Fine lymphatic networks appeared uniformly in the connective tissue beneath the perimetrium of the uterine corpus, converging into the collecting lymphatics of the cervix (Fig. 5a), and then passing laterally in the parametrium (Fig. 5b). These networks with rich anastomoses possessed obvious valve-like structures and numerous blind-ends. Larger lymphatics were often curled to form a cork-screw and represented their typical string of beaded appearance (Figs. 1d, 5a and b).

\section{Lymphatics in the Gestational Uterus}

Initial lymphatics in the endometrium were more easily detected and sometimes filled with lymph, although they were limited to the spongy layer, separated from trophoblasts. In the endomyometrial interface and myometrium, lymphatics were well developed and greatly enlarged, in contrast to those of the nulliparous monkey (Fig. 6, a, b and c). Lymphatic endothelium with high 5'-Nase activity and with notable protru- 


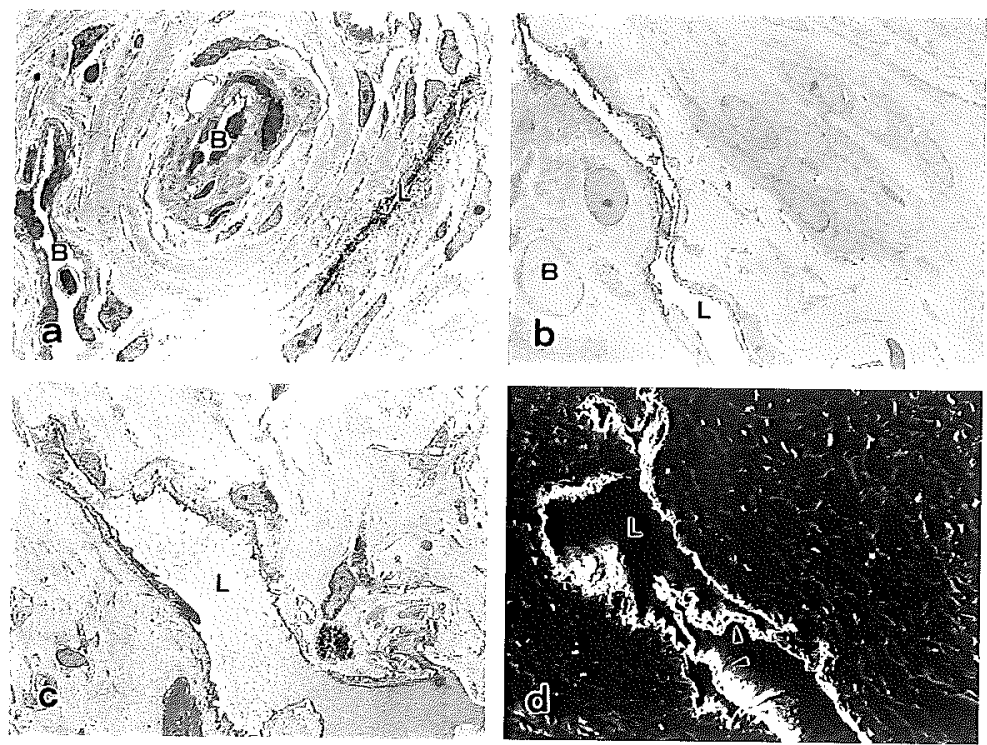

Fig. 4 In the non-gestational myometrium, the lymphatics vary from narrow and barely detectable during the early proliferative phase to large and filled with more concentrated lymph during the secretory phase. L: lymphatics; B: blood vessels

a. 5'-Nase-positive initial lymphatic with a narrow, slit-like lumen is obviously distinguished from arteriole and venule in the early proliferative phase. $(\times 1,300)$

b. In the mid-late proliferative phase, 5'-Nase reactive precipitates are evenly distributed in the luminal and basal aspects of the lymphatic endothelium. $(\times 1,500)$

c. A lymphatic with high 5'-Nase activity is filled with rich lymph in the secretory phase. $(\times 1,100)$

d. A Backscattered Electron Image (BEI)-SEM micrograph of the collecting lymphatic. Note the threedimensional two-flap valves (arrowheads), visualizing as a strong highlight. ( $\times 470)$
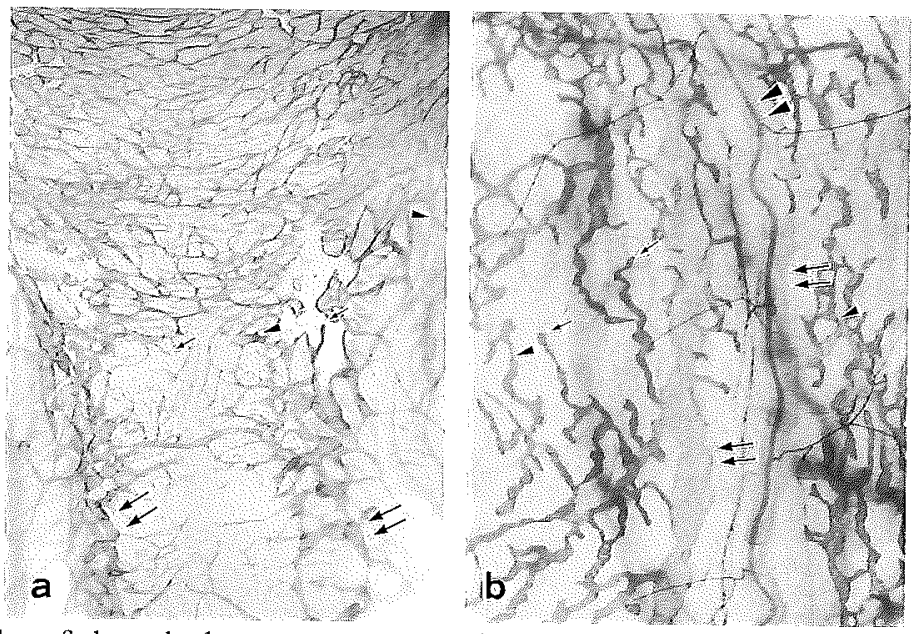

Fig. 5 Photomicrographs of the whole mount preparations showing lymphatic networks in the perimetrium with 5'-Nase staining (a) and in the mesometrium near the lateral border of the uterus with 5'-Nase-ALPase double staining (b). Strong 5'-Nase reactive product appears as a dark-brown precipitate of lead sulfide on the lymphatics, whereas that for ALPase activity is restricted to the blood vessels which are coloured blue. These lymphatic networks with rich anastomoses are characterized by numerous blind-ends (arrows) and obvious valve-like structures (arrowheads).

a. Fine lymphatic networks are evenly distributed beneath the perimetrium of the uterine corpus and drained into collecting lymphatics of the cervix (double arrows). $(\times 10)$

b. Large collecting lymphatics (double arrows) with lower 5'-Nase activity are usually flanked by ALPasepositive blood vessels (double arrowheads). ( $\times 15)$ 

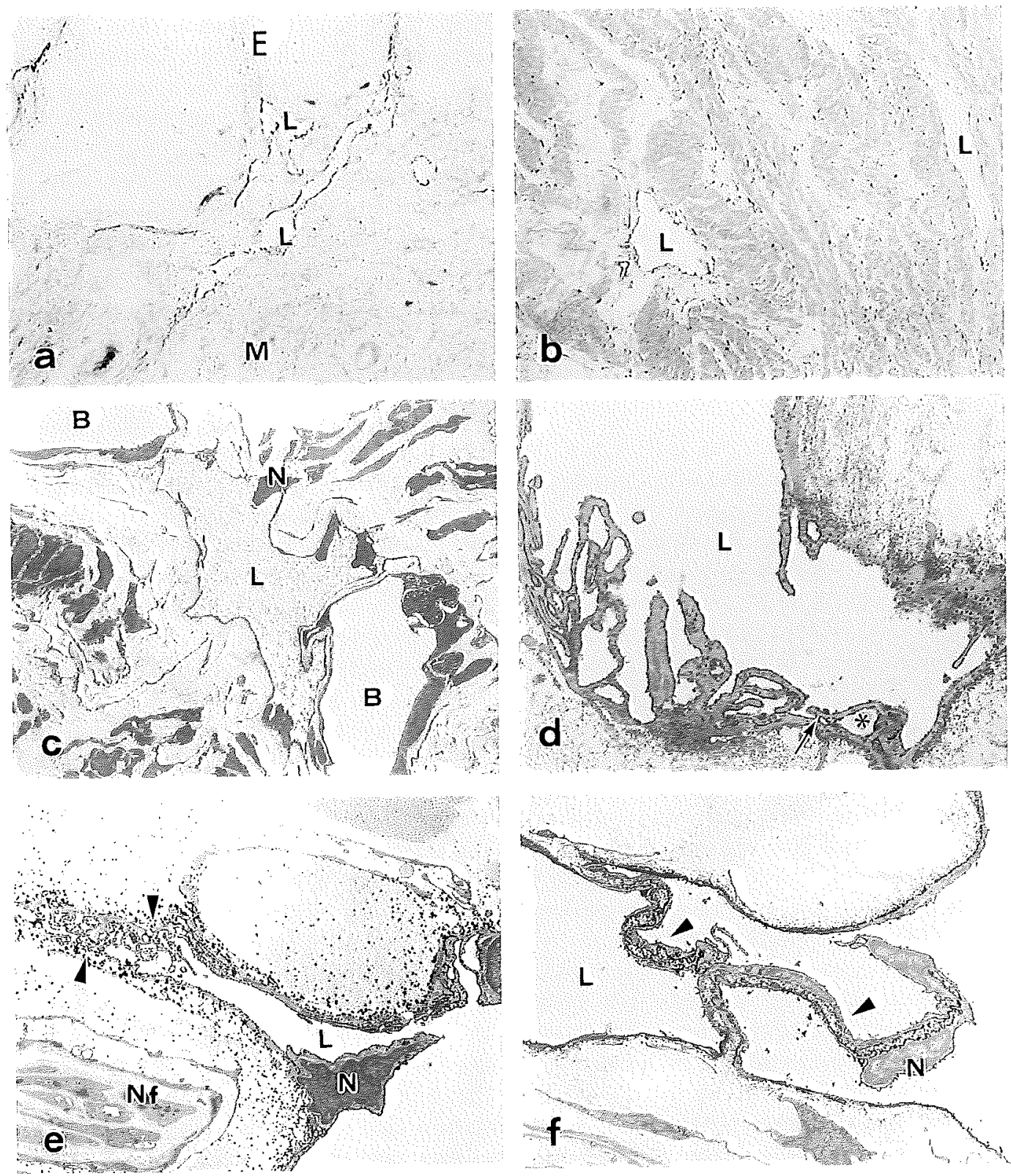

Fig. 6 In the gestational uterine wall, well developed 5-Nase-positive lymphatics showing greatly enlarged outlines under the light microscopy $(\mathrm{a}, \mathrm{b})$ and more complex structures in the endothelium than those in the non-gestational uterine wall under the electron microscopy (c, d, e, f). L: lymphatics; B: blood vessels; $N$ : nuclei of the lymphatic endothelia; Nf: nerve fiber (unmyelinated)

a. Two accompanying irregular lymphatics run at the interface between the endometrium (E) and myometrium $(\mathrm{M}) .(\times 35)$

b. In the myometrium, lymphatics running in the muscular layer show a dilated profile, in contrast to those (1b) of the nulliparous monkey. $(\times 35)$

c. A lymphatic is filled with rich lymph fluid in the endo-myometrial interface. $(\times 1,100)$

d. The lymphatic endothelium with strong 5'-Nase activity in the basal endometrium shows obvious protrusions into the luminal aspect. An intraendothelial channel (asterisk) represents a latent opening towards luminal side (arrow) and its inner surface shows even distribution of reactive precipitates. $(\times 9,500)$ e. Complex intercellular junctions (arrowheads) are also shown in the myometrial lymphatic. The junctional crevices are lined by $5^{\prime}$-Nase reactive product. $(\times 4,600)$

f. Typical two-flap valves (arrowheads) with high 5'-Nase activity are visualized in the lymphatic between the circular and longitudinal muscular layers. $(\times 2,800)$ 
sions showed typical overlapping, interdigitating intercellular junctions and intraendothelial channels, especially in the basal endometrium. The junctional crevice and inner surface of the channel were lined by 5 '-Nase reactive granules, which were occasionally scattered into neighbouring connective tissue (Fig. 6, d and e). A few open junctions occurred both in the endometrium and myometrium. Typical two-flap valves with strong 5 -Nase activity were visualized in the lymphatics between the circular and longitudinal muscular layers (Fig. 6f).

\section{DISCUSSION}

The topography and fine structure of the intrinsic uterine lymphatics in the monkey during the sexual cycle were achieved by an integrated light and electron microscopic study with enzymehistochemical method. In some previous studies, the main reason that uterus can serve as an immunologically privileged site has been assumed to be its lack in a major lymphatic supply in the endometrium (17), which contributes to the survival of the fetus as an allograft upon the mother. Investigations were likewise done on the endometrial lymphatics in mammalia and humans $(2,27)$, in which the presence of superficial lymphatics was supposed to take part in resorption rather than shedding of the endometrium during menstruation. This lymphatic contribution to endometrial growth seems to explain the result obtained in oophorectomized animals that slow withdrawal of estrogen and progesterone leads to a regression and absorption of the endometrium without bleeding (15). The limited and disputed knowledge about the uterine lymphatics in various species is based on difference in methods used. It should be noted that, with conventional histological fixation and staining techniques, the details of the endometrial lymphatic system are extremely difficult to visualize and may have easily been overlooked (2). The present findings reveal that the lymphatics with relatively lower 5 '-Nase activity are quite sparse and small in diameter in the endometrium. In contrast, rich lymphatic networks appear at the endomyometrial interface, providing a fast conducting pathway from the endometrium to the myometrium where collecting lymphatics with many valves run between the muscle bundles. The observation that initial lymphatics extend into the muscle layers along with finer, radiating connec- tive tissue septa suggests that these lymphatics also participate in lymph formation, especially in the cyclical estrogen-induced edematous condition. Initial lymphatics primarily occurring in the middle endometrium, therefore, find their way into myometrial ones and then into perimetrial ones. Perimetrial lymphatics, similar to the subserosal ones occurring in other viscera, form an important route for extraorganic lymph drainage, as we indicated before (9). However, there is considerable distinction between individual monkey in the arrangement, size and shape of the lymphatics.

The characteristic variations in the structure of the lymphatics are demonstrated in the separate phases (proliferative, secretory and menstrual) of the sexual cycle. The newly forming and growing initial lymphatics become visible in the early proliferative phase, which are characterized by extremely attenuated wall with simple intercellular junctions, accumulation of some cytoplasmic organelles and absence of surpporting fibrous framework, as the structural buildup of the endometrium begins. Toward the latter half of the proliferative phase, the lymphatics become larger in size and more complex in structure. Thus, the lymphatics in both the endometrium and myometrium vary from large and filled with more concentrated lymph during the secretory phase to narrow and barely detectable during the early proliferative phase. The data here strongly supports the standpoint that intrauterine lymphatics are the main route for migratory lymphoid cells as well as for clearance of surplus edematous fluid (18), in view of the degree of dilatation of the lymphatics correlated well with the macroscopic size of the uterus, the amount of stromal edema, and the appearance of open junction. Still the most striking finding was that initial lymphatics in the middle endometrium are affected by sporadic episodes of cessation of blood supply, exhibiting a partial destruction of endothelial wall during the course of menstruation. With breakdown products of epithelium and other endometrial components, they may be consequently expelled into uterine canal. In case the lymphatics are involved in the process of endometrial remodeling during menstruation (2), it may occur in the basal layer rather than superficial layer.

The lymphatic changes occurring in the gestational uterus are not only in the endometrium but also in the myometrium in some degree. 
During pregnancy, initial lymphatics increase greatly in number and size in the endomyometrial interface and myometrium. They represent very strong 5'-Nase activity and high endothelial permeability. Numerous open junctions and intraendothelial channels of the lymphatic endothelium may constitute a major thoroughfare for transporting interstitial tissue fluid. The result is consistent with the investigation that the flow of uterine lymph increases during pregnancy (6). Adaptive-compensatory rearrangements of the uterine lymph circulatory bed during pregnancy reveal that once a hemochorial relation between the mother and fetus is established, the continuous growth of the volumetric density and diameter of lymphatics may be connected with intensive growth of the fetus and prompt modification of the tissue metabolism.

The author is indebted to Mr. T. Kajiwara and Mrs. M. Maki for their photographic assistance.

Received I October 1998; and accepted 3 December 1998.

\section{REFERENCES}

1. Abdel Rahim S.E.A. and Bland K.P. (1985) The lymphatic drainage of the cranial part of the sheep's uterus and its possible functional significance. J. Anat. 140, 705709.

2. Blackwell P.M. and Fraser I.S. (1981) Superficial lymphatics in the functional zone of normal human endometrium. Microvas. Res. 21, 142-152.

3. CiCMANeC J.L. (1972) Lymphatic and blood vascular relationships of the uterus and ovary in the ewe. Biol. Reprod. 7, 120-121.

4. Fabian G. (1981) The cyclical changes in the uterine lymphatics of the pig. Investigations on the perimetrium. Lymphology 14, 17-23.

5. Head J.R. and Seelig JR L.L. (1984) Lymphatic vessels in the uterine endometrium of virgin rats. J. Reprod. Inmunol. 6, 157-166.

6. Hein W.R., Shelton J.N., Simpson-Morgan M.W., Seamark R.F. and Morris B. (1988) Flow and composition of lymph from the ovary and uterus of cows during pregnancy. J. Reprod. Fert. 83, 309-323.

7. JeLíneK K. (1975) Das innere Lymphgefässesystem der Gebärmutter der Kuh. III. Lymphkapillaren des Endometriums. Anat. Anzeiger: 138, 296-306.

8. JI R.C., Kato S., Mivra M. and Usui T. (1996) The distribution and architecture of lymphatic vessels in the rat stomach as revealed by an enzyme-histochemical method. Okajimas Folia Anat. Jph. 73, 37-54.

9. JI R.C. and KAto S. (1997a) Enzyme-histochemical study on postnatal development of rat stomach lymphatic vessels. Microvas. Res. 54, 1-12

10. JI R.C. and Kato S. (1997b) Demonstration of the intralobular lymphatics in the guinea pig pancreas by an enzyme-histochemical method. J. Anat. 191, 15-22.
11. Ji R.C., Kato S., Miura M., Shimoda H., Kudo T. and Usul T. (1998) Morphological characteristics of the intramural lymphatics in the collapsed and distended urinary bladder of the monkey. Lymphology 31 (Suppl), 61-64.

12. Kato S. and Mirauchi R. (1989) Enzyme-histochemical visualization of lymphatic capillaries in the mouse tongue: Light and electron microscopic study. Okajimas Folia Anat. Jpn. 65, 391-404.

13. Kato S. and Gотон M. (1990) Application of backscattered electron imaging to enzyme histochemistry of lymphatic capillaries. J. Electron Microsc. 39, 186-190.

14. Lindner H.R., Sass M.B. and Morris B. (1964) Steroids in the ovarian lymph and blood of conscious ewes. J. Reprod. Fertil. 30, 361-376.

15. Marke J.E. (1950) The relation of blood flow to endometrial growth and the inception of menstruation. In Menstruation and its Disorders (ed. Engle, E.T.) Thomas, Springfield, III, pp. 165-185.

16. Mayahara H., Hirano H., Saito T. and Ogawa K. (1967) The new lead citrate method for the ultracytochemical demonstration of activity of non-specific alkaline phosphatase (Orthophosphoric mononuclear phosphohydrase). Histochemie 11, 88-96.

17. MClean J.M. and SCOthorne R.J. (1970) The lymphatics of the endometrium in the rabbit. J. Anat. 107, 39-48.

18. Otsuki Y., Maeda Y., Magari S., Kubo H. and Sugimoto O. (1990) Lymphatics, intraepithelial lymphocytes and endometrial lymphoid tissues in the rabbit uterus: an electron microscopic and immunohistological study. Lymphology 23, 124-134.

19. Pow C.S.T. and MARTIN L. (1995) Ovarian and uterine lymphatic drainage in Australian flying-foxes (genus Pteropus, suborder Megachiroptera). Cell Tissue Res. 280, 37I-38I.

20. Robinson J.M. and Karnovsky M.J. (1983) Ultrastructural localization of 5 -nucleotidase in guinea pig neutrophils based upon the use of cerium as capturing agent. $J$. Histochem. Cytochem. 31, 1190-1196.

21. ROZHDESTVENSKY E.V. (1958) Lymphatic vessels of the mucous membrane of the uterus and their changes during the menstrual cycle. Akushersto I. Ginekologia 34, 76-80.

22. Toroski J.G. and GILL III T.J. (1977) The production of migration inhibitory factor and reproductive capacity in allogeneic pregnancies. Am. J. Pathol. 88, 333-343.

23. UfKI M. (1991) Histologic study of endometriosis and examination of lymphatic drainage in and from the uterus. Am. J. Obstet. Gynecol. 165, 201-209.

24. Vetter W. (1970) Alkalische Phosphatasen in Mastzellen, Blut- und Lymphgefäßen der Rattenzunge: 5'-Nucleotidase-, unspezifische Alkalische Phosphatase- und Polyphosphatase(ATP'ase) Aktivität unter besonderer Berücksichtigung des pH. Z. Anat. Entwickl.- Gesch. 130, 153-176.

25. WACHSTEIN M. and Meisel E. (1957) Histochemistry of hepatic phosphatase at physiologic pH. Am. J. Clin. Pathol. 27, 13-23.

26. Wermer J.A., Schünke M. and Tillmann B. (1987) Histochemical visualization of lymphatic capillaries in the rat: A comparison of methods demonstrated at the posterior pharyngeal surface. Arch. Histol. Jpn. 50, 505-514.

27. Wislocki G.B. and Dempsey E.W. (1939) Remarks on the lymphatics of the reproductive tract of the female rhesus monkey (Macaca mulatta). Anat. Rec. 75, 341-363. 